\title{
A Critical Review of Current Crisis Simulation Methodology
}

\author{
Hakkyong Kim \\ Department of Police Science \\ Korea National Police University, Gyeonggi-Do, Korea \\ Julak Lee \\ Department of Protection \& Security Administration \\ Kyonggi University, Gyeonggi-Do, Korea
}

\begin{abstract}
This paper is concerned with simulation exercises used to train key response agencies for crisis situations. While 'multi-agency' simulations are increasingly acknowledged as a necessary and significant training tool for emergency response organisations, many current crisis simulations are still focused on the revision of existing response plans only. However, a crisis requires a rapid reaction, yet in contrast to an 'emergency', the risks for critical decision makers in crisis situations are difficult to measure, owing to their illstructure. In other words, a crisis situation is likely to create great uncertainty, unfamiliarity and complexity, and consequently should be managed by adaptive or second order expertise and techniques, rather than routine or structured responses. In this context, the paper attempts to prove that the current practices of simulation exercises might not be good enough for uncertain, unfamiliar, and complex 'crisis' situations, in particular, by conducting case studies of two different underground fire crises in Korea (Daegu Subway Fire 2003) and the UK (King's Cross Fire 1987). Finally, it is suggested that the three abilities: 'flexibility', 'improvisation' and 'creativity' are critical in responding to a crisis situation.
\end{abstract}

Keywords: Underground Fire, Socio-technical Crises, Simulation, Crisis Management, Flexibility, Improvisation, Creativity.

\section{INTRODUCTION}

At disaster scenes, emergency services are required to work together to minimise the consequences of disasters as no single agency can have all the skills and resources necessary. In this context, this paper stresses the need to implement multiorganisational crisis simulations, as a means of promoting crisis preparedness. However, it appears that most current simulation training still emphasises on revision of existing response plans with a focus on testing and validating the plans. This point, as will be suggested in the ensuing sections, is in need of reevaluation. With this aim in mind, this paper firstly explores two different underground fire crises: the King's Cross Underground Fire (in the UK) and the Daegu Subway Fire (in Korea), which suggest that crises can escalate by reason of the unpredictable interplay between technical, organisational and cultural systems. Secondly, the study reviews current debates about concepts of 'emergency', 'crisis', and 'disaster', and discusses some limitations of current simulation training practice in terms of crisis management. Finally, it suggests the practical implications of crisis simulations to better deal with a crisis, focusing on crucial capabilities such as 'flexibility', ‘improvisation’, and ‘creativity’.

\section{NORMAL ACCIDENTS \& MAN-MADE DISASTERS}

In this paper, 'Normal Accidents' and 'Man-Made Disasters' theories are considered to be of relevance to analyse the two underground fire crises. Perrow in his book 'Normal Accidents (1999)' argues that a system accident or a normal accident originates from the complex and unexpected interaction of multiple technical failures [32]. He classifies 'systems' into four levels of increasing aggregation: unit, parts, subsystems, and system, and accidents are connected with damage to subsystems or the system as a whole, disrupting the ongoing of future output of the system. The causes of disasters are usually ascribed to operator errors or human errors, but he argues that the operators of the system are rather scapegoats or victims, as their errors have been already 'designed' into the complex systems. He says that modern technologies are now so complex and tightly coupled that accidents are no longer unavoidable, irrespective of operators' errors, and for this reason, he refers to accidents as 'normal accidents'.

\footnotetext{
* Corresponding author. E-mail : julaklee@hanmail.net

Manuscript received Jan.26, 2011 ; accepted Mar.20, 2011
} 
"If interactive complexity and tight coupling - system characteristics - inevitably will produce an accident, I believe we are justified in calling it a normal accident, or a system accident. The odd term normal accident is meant to signal that, given the system characteristics, multiple and unexpected interactions of failures are inevitable.” [32]

Whilst Perrow's discourse concerns tight and loose coupling in complex systems, Turner in his book 'Man-Made Disasters (1978)' emphasises the influence of an organisation culture on ignorance to hazards and signals of danger [45]. He further argues that accidents within organisations stem from not only technological factors, but also a combination of human, organisational, and cultural problems, contending that

“...it is better to think of the problem of understanding disasters as a socio-technical problem with social organisation and technical processes interacting to produce the phenomena to be studied.” [45]

Turner [45] has also suggested a six-staged model of how disasters occur in an organisation, showing the mutual dependency of both social and technical aspects of a system. At the first stage, the system operates on an accepted set of norms, values, and beliefs (operating culture). During the second stage (the incubation period), the system functions with minor problems and events arising, but these are not treated seriously as they do not fit in with the organisation's world-view of a hazard. In the third stage, a precipitating event happens, which is serious enough to provoke the awareness of decision-makers involved in the second stage. They attempt to respond to problems on the basis of preconceived ideas about the system's mode of operation. However, the system may fail to respond to these interventions, subsequently leading to stage four - system failure or breakdown. The fourth stage is the onset of disaster, which produces the fifth stage, rescue and salvage. In the sixth stage, those responsible for operating the system learn to accept what has happened. They normally carry out an 'inquiry process' with a view to establishing its causality, and subsequently, 'cultural re-adjustment' takes place in order to improve the safety of the system.

Synthesising the 'Normal Accidents' and 'Man-Made Disasters' theories, a majority of disasters seem to be associated with not only the evolution of socio-technical systems, but also the complex and tightly coupled interaction of those systems. Organisations are often confronted with failure of socio-technical systems, where decision makers are required to cope with a matter of technological risk as well as more basic types of uncertainty produced by lack of knowledge and information, or ignorance to risk [33]. Under these circumstances, 'normal accidents' originate from an unexpected combination of these socio-technical system failures, which often makes them unavoidable.

\section{KING'S CROSS UNDERGROUND FIRE}

On the evening of 19 November 1987 at around 19:30, a passenger reported a small fire on a Piccadilly Line wooden escalator at the King's Cross underground station in London [15]. One of the staff on duty went to examine the small fire. However, he had not received any fire training, and as a consequence, he did not notify either the station manager or the line controller of the fire. At that time, there were two police officers on patrol in the station, but as their radios did not work below ground, one officer ran to the surface to alert the London Fire Brigade at 19:34. Shortly before 19:40, the police decided to evacuate the station, and requested the London Underground line controllers pass trains without calling at the King's Cross station. Passengers were then evacuated upward from the lower levels of the station via the Victoria line escalators and through the tube lines ticket hall. With the benefit of hindsight, it is argued that it would have been better if they had moved passengers on the platforms by underground train to adjacent stations [12]. However, the police seemed to take correct and proper action in accordance with their existing protocol, which apparently works in the surface geographical context, but not in the surroundings below ground [4].

The first fire fighters reached the tube lines ticket hall of King's Cross station about 19:43, yet this was too late. Between 19:30 and 19:45, no one tried to extinguish the fire [15], and it quickly spread into a flashover, engulfing the whole ticket hall with flame and smoke. The flashover was later identified as an unexpected phenomenon exacerbated by the 'Trench Effect', which was brought about by combining three factors: 'the slope of the escalator', 'the trench profile which affected the later movement of air and hot combustion products', and 'the presence of flammable materials on the floor and sides of the trench' [29]. The fire was finally taken under control at 21:48, but 31 people had been killed.

The pubic inquiry [15] drew a total of 157 recommendations from the problems in relation to escalators on the underground, the response of underground staff and its training, liaison between the emergency services, and the management of safety [16]. Some of the key problems and recommendations are summarised below.

\section{Escalators on the Underground:}

"It was originally intended that the water fog equipment should be operated for a short time every night... However, experience showed that this practice caused excessive and unacceptable corrosion of the machine.... the water fog equipment has not been operated regularly." [15]

The Trench Effect:

"The demonstration, by computer simulation and fire modelling, of a 'trench effect' has shown that a mechanism exists for a fire within the escalator trough to develop very rapidly indeed. This is a newly discovered phenomenon, not previously identified in any previous fire situations or tests and not anticipated even in expert circles.” [15]

The Response of Underground Staff, and Training: 


\begin{abstract}
"Since no one had been killed in the earlier fires, they genuinely believed that with passengers and staff acting as fire detectors, there would be sufficient time to evacuate passengers safely. But they had no system to train staff in fire drill or evacuation and their attitudes towards fire gave the staff a false sense of security.” [15]
\end{abstract}

\section{The Response of the Emergency Services:}

“...there ought to be joint exercises between the emergency services, because...if such joint exercise had taken place, communications would have been better and some of the problems which presented themselves would not have proved as difficult as they did on the night.” [15]

In brief, it can be argued that the fire at the King' Cross underground was caused by an unexpected combination of technical, human, organisational, and cultural systems. Before the King's Cross fire, there had been two previous fires on wooden escalators at Green Park underground station. However, London Underground failed to learn the lessons from them [43]. This indicates that the safety culture of London Transport staff did not view fire as a legitimate threat [4]. In other words, previous fires at the London Underground did not seem to fit with the organisation's world-view of a hazard. This point also complies with Turner's second stage, the incubation period. Additionally, it appears that the newly discovered phenomenon 'Trench Effect' was a by-product of interactive complexity and tight-coupling - i.e. an unexpected mechanism of three major elements: the slope of the escalator, the trench profile, and flammable materials. Each by itself would not have caused the trench effect, but together they created it.

\section{DAEGU SUBWAY FIRE DISASTER}

In the morning of the $18^{\text {th }}$ of February 2003 at 09:52:55, a passenger with a mental and physical illness threw a plastic bottle full of gasoline, which ignited inside a car of the 1079 subway train, which stopped at the Jungangno subway station in Daegu, the third largest city in Korea [31]. The fire began to spread to all of the six carriages in the 1079 train within 2 minutes owing to the highly flammable furnishings within the train. Simultaneously, the fire alarm went off at the control centre at 09:53:12, but the subway staff on duty at the control centre ignored initial fire alarms on account of its frequent malfunctions. Meanwhile, the 1079 train driver first attempted to extinguish fire, but failed. He subsequently escaped from the train at 09:54:40, without informing the control centre of the fire [27]. At 09:54:40, the first call to the Daegu fire department came from a male passenger inside the 1079 train via his mobile phone, but there was no communication between the fire department and the control centre. At 09:55:30, a member of the staff within the station alerted the control centre to the fire, yet the centre still did not report the fire to the Daegu fire department [27]. Meanwhile, the 1080 train was approaching the Jungangno station in the opposite direction. At 09:55:40, the 1080 train driver was notified of a fire at the Jungangno station by the control centre, but he received no instructions except a warning that he should be careful in operating the train since there was a fire [24].

At 09:56:40, the 1080 train finally entered the station, and stopped alongside the blazing 1079 train. At 09:56:50, the fire wall, originally designed to protect the underground shopping malls from a fire, was automatically operated. However this made it difficult for the 1079 passengers to escape from the station [24]. For this reason, many fatalities occurred on the subway stairs, where they were presumed to be suffocated to death as they tried to escape. At 09:57:07 shortly after the 1080 train arrived, its automatic fire detector, initially intended to cut off electricity in the event of an electric short-circuit, also shut down the power supply to the 1080 train unexpectedly, preventing it from departing. At 09:57:32, the 1080 train driver communicated with the control centre about evacuation alternatives, saying "It's a mess. It's stifling. Please, take some measures. Do I have to evacuate passengers? What should I do?” [44]. However, control centre staff could not decide on the appropriate plans, and the driver simply waited for resupply of electricity without taking action. The reason for this is that train operators were not permitted to exercise their discretion and take action in case of a fire but were obliged to wait for instructions or orders from the control centre [24].

In the meantime, the 1080 train driver made three announcements between 09:57:32 and 09:58:28, asking passengers to remain seated inside a subway car although it was on fire. However, it appeared that he took right and proper action paradoxically, given the then emergency guide, "It is safer to stay on the train than attempting to get off in an emergency" [44]. The fire at last engulfed all six coaches of the 1079 train, and subsequently, at 09:58:28 spread to the adjacent 1080 train. At 09:59:00, the communication between the 1080 train driver and the control centre was cut off. Through a mobile phone, he was finally advised to kill the engine and flee from the scene by the control centre. At 10:02:00, he removed the master control key and escaped from the train, shutting down the onboard batteries that powered the train doors. This action had serious ramifications, and led to passengers being trapped within the car. The fire was finally extinguished around 13:38:00, and killed 192 people and injured 148 in total. Of 192 people, at least 142 people were killed in the 1080 train. Noticeably, the fatalities all occurred within 15 minutes or less from the onset of the fire. At 09:57:32, the fire brigade arrived at the scene, but they could not go into the station because of the heavy black smoke and toxic gases [19]. The arsonist named Daehan Kim was arrested about two hours after the fire was taken under control. He was convicted of arson and murder, and sentenced to life imprisonment. Nine subway officials including the two train drivers were also arrested, and convicted for murder through negligence, and the 1080 train driver was sentenced to 5 years of imprisonment [9].

In summary, the Daegu subway fire crisis might be argued to be caused by a complex and tightly coupled combination of technical, human, organisational and cultural factors. Such functioning of computerised control systems seems to bolster Perrow's argument that interactive complexity and tight coupling inevitably will produce a normal accident. Given that train drivers were not permitted to exercise their discretion and take action in case of a fire and had to follow the orders of the 
control centre, the 1080 train driver appeared to be victimised or scapgoated as his errors had been already 'designed' into the complex computerised systems. In addition, the safety culture of the control centre seemed to play a vital role in ignoring fire alarms on account of previous malfunctions. Before the Daegu subway fire in 2003, there had also been four major incidents during the construction of the Daegu Metropolitan Subway Line, where in total 108 people had been killed [44]. Therefore, it can be concluded that the seed of the Daegu subway fire crisis may have been sown or incubated several years even before its onset, although it was triggered by the arson attack.

\section{CRISIS AS TWO SIDES OF A COIN: UNCERTAINTY AND OPPORTUNITY}

What are the definitions of 'emergency', 'crisis' and 'disaster'? While there have been many attempts to suggest clear definition, there is no generally accepted terminology [16]. For the distinction, Borodzicz' doctoral thesis [4] provides a logic and effective exemplar. He argues that there needs to be a clear distinction between the concepts of 'emergency', 'crisis', and 'disaster', and understanding this difference can contribute to improving the design and implementation of crisis simulations.

He defines an 'emergency' as a 'situation requiring a rapid and highly structured response where the risks for critical decision makers can to a relative degree be defined' [4]. Therefore, emergency services are able to manage 'emergencies' by deploying their established and structured routines, skills and procedures [46]. A 'crisis' is defined as a 'situation requiring a rapid response (for this reason they are all too easily misconceived as emergencies), although in contrast, the risks for critical decision makers are difficult to define owing to ill-structure' [4]. In this context, a crisis can be characterised by three dimensions such as unexpected threats, uncertainty and a need for immediate decision-making [37] [3]. A crisis situation is likely to create great uncertainty, unfamiliarity and complexity, and consequently can be managed by adaptive or second order expertise and techniques, not routine or structured responses [46] [36].

However, despite such uncertainty and difficulty, most importantly, its successful management can transform a crisis situation into a better one, and conversely its mismanagement can easily lead to the next crisis [40]. In other words, there is still an opportunity for good management or organisational change in crisis situations [5] [38]. The interesting point here is that the English word, 'crisis' stems from the Greek 'krisis', which is synonymous with making a right judgment when it is timely to decide. So in this respect, a crisis can be translated as an opportunity (i.e. a right time) for decision [30] [1]. Another well-known analogy here is that the Chinese word for crisis is comprised of two different characters: the first character (危) symbolises a 'danger (risk)', and the second one (機) represents an 'opportunity' [13] [6]. Hence, in the Chinese context, a crisis is seen as another opportunity. Also, the French word for crisis is defined (in medical terms) as a 'sudden variation during a disease which determines the outcome for better or for worse' [17].

In contrast, a 'disaster' is defined as a 'cultural construction of reality' [14]. Put simply, a disaster means a stage where harm has been caused and there is an element of damage limitation because of a failure to deal with the aforementioned emergencies or crises. The central argument here about a disaster is that it also consist of ongoing emergencies and crises, and hence is often considered as the most difficult phenomenon to successfully manage. Crisis or disaster produces a different social order (non-routine events), and they can not be handled by the same organisational structures and skills used to deal with emergencies [20] [35]. However, it should be also borne in mind that 'emergency', 'crisis' and 'disaster' can be understood differently by different organisations involved. There are different organisational, social and cultural constructs of the definitions, and this subjective nature appears to cause the same event to be interpreted differently.

\section{CRISIS SIMULATION PARADOXES}

Crisis simulations are now considered as an integral part of the emergency planning. However, the problem is that the purpose of such crisis simulations is still restricted to training people to apply procedures and plans to become familiar with necessary skills and locations [21]. In other words, most of the crisis simulations in emergency services seem to concentrate on developing agency specific expertise, increasing familiarity or obtaining experience in specific task functions [11]. For instance, the UK Cabinet Office [8] says that exercises have three main purposes: validating plans (validation), developing staff competencies and giving them practice in carrying out their roles in the plans (training), and testing well-established procedures (testing).

In a 'crisis' where decision-makers are thrown into a concrete reality of physical danger by such ill-structure, current practice in the UK, which emphasises the application of existing plans and procedures, might not be sufficient to deal with a crisis. In this context, several questions can be raised as to why current simulations might be inadequate to manage crises; firstly, a crisis by definition creates uncertainty, and ambiguity, and as a result, the 'planning paradox' might happen whereby the more elaborate a crisis plan is, the less it will be used in a crisis as it necessarily must focus on a narrow range of possible threats [28]. Furthermore, it is not possible to predict and stipulate every possible emergency scenario and script every response to radically changing situations [48]. Emergency services appear to successfully deal with emergency situations similar to scenarios for which they are trained, but how can they deal with unexpected situations which they are not prepared or trained for?

Secondly, contingency planning demands that threats are ordered by importance and are coherent, yet crises are not subject to such predictable deconstruction. Crisis simulations are an integral part of contingency planning, and a starting point for this is to decide emergency scenarios, assisted by quantitative probabilistic risk analysis (e.g. what is the most likely?). However, the quantitative probabilistic risk assessment might produce the 'open systems paradox' [42]. A 
crisis is an 'open system', where there are numerous equally but different possible trajectories by which a crisis can develop into a disaster. In contrast, quantitative risk assessment is a 'closed system', where a finite number of failure modes for each hypothetical situation are derived. Accordingly, 'worst case' scenarios are seldom taken into account, although a valuable exercise in preparing for a crisis need to work on them [34].

Thirdly, crisis simulations serving as a validation or testing tool for emergency plans might often create the institutional blind spots around the processes of crisis response. One advantage of the simulations is that they can create a 'mistakefriendly' learning environment, encouraging trial-error learning to take place [25] [39]. However, no one wants to make mistakes in crisis simulations if they are synonymous with a test. Mistakes would be synonymous with failure, and participants may be under pressure to demonstrate their success - a phenomenon which would contradict the purpose of trialerror learning. Exercises are settings where failure should be allowed and even encouraged to a certain extent. Otherwise, emergency services might lose the opportunity to learn the limits of their own, and their organisations' abilities in this type of simulation.

Finally, any difference in behaviour is judged to be a violation of rules, and can be sanctioned in the case of simulations as an evaluation tool, whereas people often improvise their response to cope with real situations in progress. Participants are usually assessed for their strict application of plans vis-à-vis the standard procedures, and as a result, deviations from predetermined plans and procedures are usually evaluated negatively in current simulations [21]. However, in reality, when confronted with unanticipated accidents, people usually find innovation and adaptations of plans or skills to cope with them [49]. He further argues that simulation as a testing tool appears naive since it doesn't cover the uncertainty and complexity that will arise during a crisis. Adhering to operating procedures might not provide the opportunity to explore alternatives which may be needed when faced with a crisis.

\section{IMPLICATIONS OF CRISIS SIMULATION}

The King's Cross Underground fire and the Daegu subway fire reflect that simple attempts to cope with crises in a routine manner according to set procedures and practices are likely to fail. For instance, evacuating passengers upward in the underground although the fire moves upward, requiring passengers to stay on the burning train, or just waiting for the control centre's instruction led to disastrous results. However, it is not feasible to predict every single worst case scenario, stipulate its response in a plan, and test it through simulations. Too much dependence on predetermined rules and procedures might rather cause crisis simulation paradoxes. For this reason, crisis responses need to be improvised to some extent, based on circumstances, time constraint, and resources available case by case. In a similar vein, Turner [46] argues that training for a crisis requires a level of 'flexibility and improvisation', which can contribute to filling the gap between a plan and the reality of crisis. According to Turner, 'flexibility' is considered the capability to be sensitive to the changing meaning and consequences of an incident, and 'improvisation' is defined as the ability to adjust to the unexpected and new situations by creating a new paradigm which helps to guide the continuing response.

Kendra and Wachtendorf [22] further argue that improvisation requires creativity and simultaneously, creativity is a dimension of improvisation. They contend that not only flexibility but also creativity is a virtue of crisis managers. Creativity can be defined as the ability to generate new alternatives, which can accomplish fundamental objectives in ways previously unforeseen, with aspects of both novelty and effectiveness [10]. Therefore, creativity can boost the ability to improvise in new physical and social environments through generating new and novel solutions to open-ended problems that will arise in crisis situations. In other words, response plans are also subject to alter, particularly when combined with uncertainty and ambiguity of a crisis, and in this case, creativity comes to serve as a key factor to expedite improvisation through finding novel and unprogrammed solutions.

An interesting but rather surprising comparison with crisis simulations is made for martial arts practice [7]. In martial arts, at first a beginner usually practices various techniques with an imaginary opponent. Individual training of this nature enables trainees to get accustomed to the body mechanics involved in the performance of techniques. Certain optimum patterns of movement and skills must be set up, which can be only feasible if trainees repeat training drills with dedication. Then, to become proficient in martial arts, trainees must practice free style sparring with a partner, where merely doing the form in a pre-programmed manner does not work, and instead, they have to adapt and apply the skills and movement in an unprogammed manner to attack partners or avoid their attacks. Noticeably, skilled masters are proficient in delivering their techniques and skills adaptively through a combination of almost simultaneous application of defence and attack. They occasionally show their combined techniques in creative and unprogrammed ways. In this sense, crisis simulations can be compared with freestyle sparring in martial arts, where flexibility, improvisation and creativity are also of great importance.

\section{CONCLUSION}

The investigation into the two underground fire crises also demonstrated that crisis does not respect the conventional training methodology - i.e. the current practice of crisis simulations. This also raises the importance of differentiating the concepts of 'crisis', 'emergency', and 'disaster', with regard to designing and conducting crisis simulations. Current simulation practice seems to be deficient in dealing with crises, given that socio-technical crises require a new approach to crisis simulations. For instance, responding organisations need to react to unexpected accidents (crisis) in an adaptive manner as well as in creative ways. To fill the gap between the rigidity of plans and the actuality of a crisis, it is finally argued that crisis simulations demand flexibility, improvisation and 
creativity. To paraphrase what Toft \& Reynolds [41] have mentioned, we owe it to those who have lost their lives, been injured or suffered in both the King's Cross underground fire and the Daegu Subway disaster to learn the lessons and apply them to prevent similar failures in crisis response. Crisis simulations need to be designed for flexibility, improvisation, and creativity to happen, which the author believes, can contribute to solving the crisis simulations paradoxes. Without flexibility, improvisation, and creativity, indeed, there could not be a real 'crisis simulation'.

\section{REFERENCES}

[1] Banfield, Krisis or Crisis? Workplace Ministry in Hertfordshire \& Bedfordshire, Available: http://www.workplaceministry.org.uk/herts\&beds/newsle tter/Summer\%202007.pdf [Accessed: 2 November, 2008], 2007.

[2] U. Beck, Risk Society, Sage, London, 1992.

[3] A. Boin, O. 'T Hart, E. Stern, and B. Sundelius, The Politics of Crisis Management: Public Leadership under Pressure, Cambridge University Press, Cambridge, 2005.

[4] E. P. Borodzicz, Risky business: Crisis simulations examined in the context of the safety people, doctoral dissertation, University of London, London, 1997.

[5] E. P. Borodzicz and L. Van Haperen, "Individual and Group Learning in Crisis Simulations", Journal of Contingencies and Crisis Management, vol. 10, no. 3, 2002, pp. 139-147.

[6] E. P. Borodzicz, "The Missing Ingredient is the Value of Flexibility”, Simulation and Gaming, vol. 35, no. 3, 2004, pp. 414-426.

[7] E. P. Borodzicz, Risk, crisis \& security management, John Wiley \& Sons, Chichester, 2005.

[8] Cabinet Office, Emergency Preparedness, HM Government, London, 2005.

[9] J. Chung, N. Sung, W. C. Taylor, and H. Kim, "Comparative Study of Underground Fires: the Cases of the Daegu Subway Fire and the King's Cross Underground Fire”, Proc. the 84th Transportation Research Board Annual Meeting, Washington D. C., 2005.

[10] R. T. Clemen and T. Reilly, Making Hard Decisions with DecisionTools, Duxbury Thomson Learning, USA, 2001.

[11] M. T. Crichton, R. Flin, and A. R. William, "Training Decision Makers-Tactical Decision Games”, Journal of Contingencies and Crisis Management, vol. 8, no. 4, 2000, pp. 208-217.

[12] B. Crossland, “The King's Cross Underground Fire and the Setting Up of the Investigation”, Fire Safety Journal, vol. 18, no. 1, 1992, pp. 3-11.

[13] J. R. Daring, "Crisis Management in International Business: Keys to Effective Decision Making”, Leadership and Organisation Development Journal, vol.15, no. 8, 1994, pp. 3-8.

[14] W. R. Dombrowsky, "Again and Again: Is a Disaster What We Call Disaster? Some Conceptual Notes on Conceptualizing the Object of Disaster Sociology”,
International Journal of Mass Emergencies and Disasters, vol. 13, no. 3, 1995, pp. 241-254.

[15] D. Fennell, Investigation into the King's Cross underground fire, HMSO, London, 1998.

[16] R. Flin, Sitting in the Hot Seat: Leasers and team for Critical Incident Management, Jones Willey \& Sons, Chichester, 1996.

[17] C. Hansen and C. Drieu, Changing Definitions of Crisis. In Horlick-Jones, T, Amendola, A. and Casale, R. (Eds.), Natural Risk and Civil Protection, E\&FN Spon, London, 1994.

[18] Home Office, Dealing with Disasters (Revised Third Edition), Home Office, London, 2003.

[19] W. H. Hong, Investigation Report on the 2.18 Daegu Subway Accident, Kyungpook University, Daegu, 2004.

[20] T. Horlick-Jones, "Planning and coordinating urban emergency management”, Disaster Management, vol. 6, no. 3, pp. 141-146.

[21] J. M. Jacques and M. Specht, "Cognition towards crisis: the blind man held a handful of snow...and concluded that white was cold”, International Journal of Emergency Management, vol. 3, no. 1, 2006, pp. 21-39.

[22] J. M. Kendra and T. Wachtendorf, Creativity in Emergency Response to the World Trade Centre Disaster, In J. L. Monday, (Ed.), Beyond September 11th: An account of Post-Disaster Research, Natural Hazards Research and Information Centre, University of Colorado, USA, 2003a.

[23] J. M. Kendra and T. Wachtendorf, "Elements of Resilience after the World Trade Centre Disaster: Reconstructing New York City's Emergency Operation Center”, Disasters, vol. 27, no. 1, 2003b, pp. 37-53.

[24] B. S. Kim, A Study on the Building-up of the Crisis Management System for the Effective Disaster management: Focus on the analysis of Dae-gu Subway fire accident, Master Dissertation, University of Gongju, Korea, 2005.

[25] W. C. Kriz, "Creating effective learning environments and learning organisations through gaming simulation design”, Simulation and Gaming, vol. 34, no. 4, 2003, pp. 495-511.

[26] C. Lalonde, "In search of Archetypes in Crisis Management”, Journal of Contingencies and Crisis Management, vol.12, no. 2, 2004, pp. 76-88.

[27] C. S. Lee, A Study on the Activities of Emergency Response Organisation: Focusing on the Case of Fire in Daegu Subway, PhD dissertation, University of Ulsan, Korea, 2007.

[28] A. McConell and L. Drennan, "Mission Impossible? Planning and Preparing for Crisis", Journal of Contingencies and Crisis Management, vol. 14, no. 2, 2006, pp. 59-69.

[29] K. Moodie, "The Technical Investigation of the Fire at London's Kings' Cross Underground Station”, Journal of Fire Protection Engineering, vol. 3, no. 2, 1991, pp. 49-63.

[30] E. Morin, "Pour une crisiologie”, Communications, vol. 25, 1976, pp. 149-163.

[31] NEMA (National Emergency Management Agency), Disaster reports: Fire in Daegu Subway, 
Available:http://www.nema.go.kr/eng/m4_subway.jsp, [Accessed: 1 December 2008], 2004.

[32] C. Perrow, Normal Accidents: Living with High-Risk Technologies, Princeton University Press, NJ, 1999.

[33] N. Pidgeon, "Safety culture: key theoretical issues", Work and Stress, vol.12, no. 3, 1998, pp. 202-216.

[34] M. Punch and G. Markham, "Policing Disasters: the British experience”, International Journal of Police Science and Management, vol. 3, no. 1, 2000, pp. 40-54.

[35] E. Quarantelli, "Disasters are different, therefore planning for and managing them requires innovative as well as traditional behaviours". Proc. the Third Emergency Planning and Disaster Management Conference, July, Lancaster University, Lancaster, 1995.

[36] U. Rosenthal, Crisis management: second order techniques, In T. Horlick-Jones, A. Amendola, and R. Casale (Eds.), Natural Risk and Civil Protection, E\&FN Spon, London, 1994.

[37] U. Rosenthal, R. A. Boin, and L. K. Comfort, The Changing World of Crisis and Crisis Management, In U. Rosenthal, R. A. Boin, and L. K. Comfort (Eds.), Managing Crises: Threats, Dilemmas and Opportunities, Springfield, USA, 2001.

[38] C. Roux-Dufort, "Is Crisis Management (Only) a Management of Exceptions?”, Journal of Contingencies and Crisis Management, vol. 15, no. 2, 2007, pp. 105-114.

[39] D. Smith, "For whom the bell tolls: Imagining accidents and the development of crisis simulation in organisations”, Simulation and Gaming, vol. 35, no. 3, 2004, pp. 347-362.

[40] P. 't Hart and R. A. Boin, Between crisis and normalcy: The long shadow of post-crisis politics, In U. Rosenthal, R. A. Boin, and L. K. Comforts (Eds.), Managing crises: Threats, dilemmas, opportunities, Springfield, USA , 2001.

[41] B. Toft and S. Reynolds, Learning from Disasters: a management approach ( $1^{\text {st }}$ edition), ButterworthHeinemann, Oxford, 1994.

[42] B. Toft, Limits to the mathematical modelling of disasters, In C. Hood and D. Jones (Eds.), Accident and Design: contemporary debates in risk management, UCL press, London, 1996.

[43] B. Toft and S. Reynolds, Learning from Disasters: a management approach ( $2^{\text {nd }}$ edition), Perpetuity Press, Leicester, 1999.

[44] TRB (Transportation Research Board), TCRP Report 86/Vloume 12: Making Transportation Tunnels Safe and Secure, Transportation Research Board of the National Academies, Washington D. C., 2006.

[45] B. A. Turner, Man-Made Disasters, Wykeham Publications, London, 1978.

[46] B. A. Turner, "Flexibility and Improvisation in Emergency Services”, Disaster Management, vol. 6, no. 2, 1994, pp. 84-90.

[47] D. Upton, Official Crisis Simulations in the UK and Elsewhere, In P. R. J. Trim and Y. I. Lee (Eds.), The International Simulation and Gaming Research Yearbook: Effective Learning from Games and Simulations, SAGSET, Edinburg, 2007.
[48] G. R. Webb and F. R. Chevreau, "Planning to improvise: the importance of creativity and flexibility in crisis response”, International Journal of Emergency Management, vol. 3, no. 1, 2006, pp. 66.-72.

[49] J. L. Wybo, The Role of Simulation Exercises in the Assessment of Robustness and Resilience of Private or Public organisations, In H. J. Pasman and I. A. Kirillov (Eds.), Resilience of Cities to Terrorist and other Threats: Learning from 9/11 and further Research Issues, Springer, Netherlands, 2008.

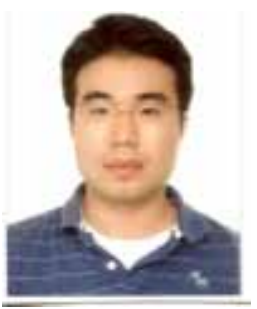

Hakkyong Kim

$\mathrm{He}$ is a professor of criminal investigation at the Korea National Police University in the Republic of Korea. He earned his MSc degree in Risk, Crisis \& Disaster Management from the University of Leicester, UK. He is currently finished with his $\mathrm{PhD}$ research on a cross-cultural comparison of simulation training between emergency services in Korea and the UK at the University of Portsmouth, UK.

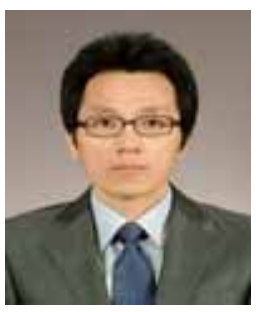

Julak Lee

He is an assistant professor of the department of protection and security management at the Kyonggi University. He obtained Master's degree from the Michigan State University, US, majoring in Criminal Justice, and earned $\mathrm{PhD}$ in Criminal Justice from the University of 